\section{Triagem para a IDENTiFicação de USO NoCivo de ÁLCOOL NA ATENÇäO PRIMÁRIA À SAÚdE}

Foi com bastante interesse que lemos o artigo "Validation of the Brazilian Version of Alcohol, Smoking and Substance Involvement Screening Test (ASSIST)", publicado na Revista da Associação Médica Brasileira'. É de grande importância a validação de novos instrumentos em nosso meio que possam auxiliar na triagem de pacientes na atenção primária à saúde que fazem uso de álcool de maneira nociva, devido à sua alta prevalência e à possibilidade de intervenção terapêutica eficaz nesse contexto.

Estudo transcultural, multicêntrico, realizado pela Organização Mundial da Saúde em pacientes da atenção primária à saúde mostrou que a prevalência de dependência ao álcool foi de $2,7 \%$, sendo o terceiro transtorno psiquiátrico mais prevalente, ficando atrás apenas de depressão e transtorno de ansiedade generalizada ${ }^{2}$. Estatísticas americanas mostram que $70 \%$ dos pacientes com quadro de dependência ao álcool foram atendidos em serviços médicos gerais nos últimos seis meses, devido aos mais variados problemas de saúde ${ }^{3}$.

No entanto, a maioria dos pacientes da atenção primária à saúde, que consome álcool em níveis de risco, não apresenta necessariamente quadro de dependência à substância. Pesquisas epidemiológicas têm demonstrado que a maior parte dos problemas ocasionados pelo uso do álcool nesses pacientes da atenção primária à saúde manifesta-se justamente entre aqueles que não são dependentes da substância, mas que apresentam comportamento de risco quando bebem, por exemplo, dirigindo embriagados. Assim, a detecção, não só dos pacientes que apresentam quadro de dependência ao álcool, mas também daqueles que fazem uso da substância de maneira perigosa, é bastante importante na atenção primária à saúde ${ }^{4}$.

O Projeto Bandeira Científica da Faculdade de Medicina da Universidade de São Paulo, que tem por objetivo ajudar comunidades menos desenvolvidas a organizar maneiras mais adequadas de lidar com seus principais problemas de saúde pública na atenção primária, está desenvolvendo no momento suas atividades em duas cidades do interior de Alagoas: Teotônio Vilela e São José da Tapera. Em um primeiro momento, realizou-se investigação para levantar aqueles que seriam os problemas mais importantes a serem equacionados. Para a investigação do uso nocivo de álcool realizou-se um rastreamento entre os pacientes utilizando-se o "Alcohol Use Disorders Identification Test" (AUDIT), instrumento desenvolvido pela Organização Mundial da Saúde ${ }^{5}$ e já validado para o uso em nosso meio ${ }^{6,7}$. Cento e noventa e cinco pacientes foram avaliados aleatoriamente, sendo 131 mulheres e 64 homens, todos acima de 16 anos de idade. No total dos pacientes avaliados, 12,82\% faziam uso de álcool de maneira nociva, sendo 2,29\% das mulheres e 34,37\% dos homens ( $<<0,00 \mathrm{I}$ ). Na população masculina, o uso nocivo de álcool foi mais comum entre os mais jovens, sendo que 40,9\% dos homens que apresentavam uso nocivo da substância apresentavam menos de 30 anos de idade. A idade média da população masculina estudada foi de 44,37 (+/- 16,42) anos, sendo que a idade do grupo que fazia uso nocivo de álcool foi de 37,27 (+/- 13,53) anos, aumentando para 48,09 (+/- 16,72) anos entre aqueles que não faziam uso nocivo $(p=0,022)$. Assim, podemos perceber que o uso de álcool realizado de maneira nociva encontrase em níveis importantes, principalmente entre os pacientes mais jovens e do sexo masculino.

Diante desse quadro, as triagens configuram-se em um método importante para a detecção de pacientes que apresentam uso nocivo de álcool, podendo ser utilizadas como precursoras de intervenções terapêuticas por médicos generalistas na atenção primária à saúde. Isso porque, intervenções realizadas pelo médico generalista na atenção primária à saúde podem melhorar o padrão de uso de álcool pelo paciente, e não diminuem sua satisfação com o tratamento clínico. Assim, o estudo de maneiras mais adequadas de se fazer a detecção de pacientes que apresentam uso nocivo de álcool, através da utilização de instrumentos de triagem, é bastante importante em nosso meio ${ }^{2}$.

\section{Quirino Cordeiro Leandro Michelon Rafael Bernardon Ribeiro Carla Kamitsuji Camila Magalhäes Silveira laura Helena Guerra Andrade São Paulo - SP}

Referências

I. Henrique IF, De Micheli D, Lacerda RB, Lacerda LA, Formigoni ML. Validation of the brazilian version of Alcohol, Smoking and Substance Involvement Screening Test (ASSIST). Rev Assoc Med Bras 2004;50: 199-206.

2. Goldberg DP, Lecrubier Y. Form and frequency of mental disorders across centres. In: Üstün TB, Sartorius N, editors. Mental illness in general in general health care: an international study. Chichester: John Wiley \& Sons; 1995. p.323-34.

3. Shapiro S, Skinner EA, Kessler LG, Von Korff M, German PS, Tischler GL, et al. Utilization of health and mental health services: three epidemiological catchment area sites. Arch Gen Psychiatry 1984;41:971-8.

4. World Health Organization. The world health report 2001 - mental health: new understand, new hope. Geneve: WHO; 2001

5. Saunders JB, Aasland OG, Bebor TF, De La Fuente JR, Grant M. Development of the Alcohol Use Disorders Identification test (AUDIT): WHO Collaborative Project on Early Detection of Persons with Harmful Alcohol Consumption II. Addiction 1993;88:791-804.

6. Mendez EB. Uma versão brasileira do AUDIT (Alcohol Use Disorders Identification Test) [dissertação]. Pelotas: Faculdade de Medicina, Universidade Federal de Pelotas; 1999.

7. Mendoza-Sassi RA, Beria JU. Prevalence of alcohol use disorders and associated factors: a population-based study using AUDIT in southern Brazil. Addiction 2003;98:799-804. 